\title{
Tests of current algebra and partially conserved axial-vector current in the subthreshold region of the pion-nucleon system
}

\author{
William B. Kaufmann \\ Arizona State University, Tempe, Arizona 85287-1504 \\ Gerald E. Hite \\ Texas A\&M University at Galveston, Galveston, Texas 77553-1675 \\ (Received 16 February 1999; published 1 October 1999)
}

\begin{abstract}
The recently available $\pi N$ elastic, phase-shift analysis SP98, and interior dispersion relations are used to obtain the invariant amplitudes in the subthreshold crescent where they can be compared directly to the predictions of chiral theories. [S0556-2813(99)05210-3]
\end{abstract}

PACS number(s): 13.75.Gx, 11.55.Fv, 11.30.Rd

\section{INTRODUCTION}

Current algebra and partially controlled axial-vector current (PCAC) make powerful predictions in the threshold and subthreshold regions of the $\pi N$ system [1]. Some of the most important examples are the Adler consistency condition [2] (which predicts a zero in the pole-subtracted isospin-even amplitude for one soft pion), the Weinberg-Tomozawa prediction of $\pi N$ scattering lengths [3], the Adler-Weisberger sum rule [4] (which constrains the isospin-odd amplitude at $\nu=t=0$ for massless pions), and the $\pi N \sigma$ term (which measures the chiral-symmetry breaking in the $\pi N$ system). The values of these quantities are determined from amplitudes evaluated within the small subthreshold "crescent'" region in the Mandelstam diagram shown in Fig. 1 [5]. Because the crescent lies below the physical threshold of all of the crossed reactions $(\pi N \rightarrow \pi N, \quad \bar{\pi} N \rightarrow \bar{\pi} N, \quad$ and $N \bar{N} \rightarrow \pi \pi)$, the invariant amplitudes are real in this region.

In this article we report on an application of a recent Virginia Polytechnic Institute phase-shift analysis SP98 [6] and interior dispersion relations (IDR's) [7] to map the relevant amplitudes within the entire crescent [8]. Hence, this analysis provides current tests of several predictions of chiral symmetry. The present work is an extension and update of work done at the beginning of the era of the meson factories [9]; since then, new high-precision $\pi N$ data have been obtained, from which improved low-energy phase shifts have been extracted. The general structure of the pion-nucleon invariant amplitudes has long been known [10], of course, but the IDR method is especially well suited for studies of the subthreshold region, especially when coupled with the high-precision VPI phase-shift analyses.

\section{IDR AND THE SUBTHRESHOLD REGION}

We will use two sets of independent variables: $(a, t)$ and $(\nu, t)$, where $\nu \equiv s-u$, and $a$ is the IDR path parameter defined by $a=-\left[s u-\left(m^{2}-\mu^{2}\right)^{2}\right] / t[7,11] . m$ is the proton mass, and $\mu$ is the charged pion mass. IDR's are "dispersed' ' in $t$ along curves of constant negative $a$, so $(a, t)$ are natural choices as independent variables. In these variables we have $\nu^{2}=\left(t-4 \mu^{2}\right)\left(t-4 m^{2}\right)+4 a t \equiv\left[t-t_{-}(a)\right][t$ $\left.-t_{+}(a)\right]$. The portion of a typical curve of constant $a$ in the neighborhood of the crescent is shown in Fig. 1. For $t<0$ the path lies entirely within the $s$-channel physical region and passes through the $s$-channel threshold point. In the interval $t_{-}(a)<t<t_{+}(a), \nu$ is pure imaginary, and for $t>t_{+}(a)$ the path lies entirely within the $t$-channel physical region.

Interior dispersion relations are written for amplitudes which are $s u$ crossing symmetric: $F(\nu, t)=F(-\nu, t)$. In terms of the variables $(a, t)$, the unsubtracted form of an IDR is

$$
\begin{aligned}
F(a, t)= & F_{N}(a, t)+\frac{1}{\pi} \int_{4 \mu^{2}}^{\infty} \frac{\operatorname{Im} F\left(a, t^{\prime}\right) d t^{\prime}}{t^{\prime}-t} \\
& +\frac{1}{\pi} \int_{(m+\mu)^{2}}^{\infty} \operatorname{Im} F\left(a, t\left(s^{\prime}, a\right)\right) \\
& \times\left[\frac{1}{s^{\prime}-s}+\frac{1}{s^{\prime}-u}-\frac{1}{s^{\prime}-a}\right] d s^{\prime},
\end{aligned}
$$

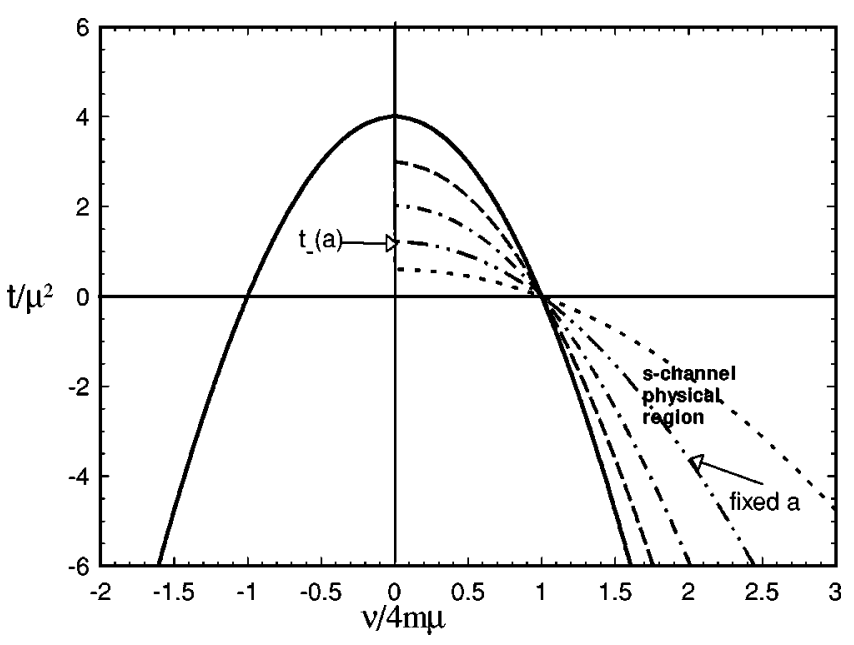

FIG. 1. Mandelstam diagram for the $\pi N$ system. The crescent is the small-lenslike region lying between the line $t=0$ and the hyperbola $s u=\left(m^{2}-\mu^{2}\right)^{2}$. Curves are shown with path parameters $a$ $=-0.3 \mathrm{GeV}^{2}$ (long-dashed line), $-0.86 \mathrm{GeV}^{2}$ (dash-dotted line), $-2.0 \mathrm{GeV}^{2}$ (dash-double-dotted line), and $-5.0 \mathrm{GeV}^{2}$ (shortdashed line). The curve with $a=-0.86 \mathrm{GeV}^{2}$ intersects the $\nu$ axis at the Cheng-Dashen point. 
or simply

$$
F(a, t)=F_{N}(a, t)+\mathcal{D}_{F}(a, t)+I_{F}(a, t),
$$

where $F_{N}$ is an appropriate IDR Born term to be defined shortly, $s=s(a, t)=\frac{1}{2}[\Sigma-t+\nu(a, t)], \quad u=u(a, t)=\frac{1}{2}[\Sigma-t$ $-\nu(a, t)], \quad t\left(s^{\prime}, a\right)=\left[-s^{\prime}\left(\Sigma-s^{\prime}\right)+\left(m^{2}-\mu^{2}\right)^{2}\right] /\left(a-s^{\prime}\right)$, and where $\Sigma \equiv 2 m^{2}+2 \mu^{2}$. The integral over the $t$-channel cut, $\mathcal{D}_{F}(a, t)$, is called the "discrepancy function." Since the data needed for the $s$-channel integral, i.e., the last term in Eq. (2), are available only up to some value $s_{\max }$, in practice one defines the data integral by

$$
\begin{aligned}
I_{F}\left(s_{\max } ; a, t\right)= & \frac{1}{\pi} \int_{(m+\mu)^{2}}^{s_{\max }} \operatorname{Im} F\left(a, t\left(s^{\prime}, a\right)\right) \\
& \times\left[\frac{1}{s^{\prime}-s}+\frac{1}{s^{\prime}-u}-\frac{1}{s^{\prime}-a}\right] d s^{\prime}
\end{aligned}
$$

and the "effective discrepancy function" by

$$
\mathcal{D}_{F}\left(s_{\max } ; a, t\right) \equiv F(a, t)-F_{N}(a, t)-I_{F}\left(s_{\text {max }} ; a, t\right) .
$$

Combining its definition with Eq. (1), $\mathcal{D}_{F}\left(s_{\max } ; a, t\right)$ is the sum of $\mathcal{D}_{F}(a, t)$ and the high-energy part of the $s$-channel integral. Both parts can be expected to vary slowly in that portion of the $s$-channel physical region where $|t|$ $\ll\left|t\left(s_{\max }, a\right)\right|$.

The effective discrepancy function defined in Eq. (4) varies slowly even if a subtracted form of the dispersion relation, Eq. (1), were required for convergence. From its definition and the subtracted version of Eq. (1), $\mathcal{D}_{F}\left(s_{\max } ; a, t\right)$ is the sum of subtraction terms (e.g., amplitudes and $I_{F}$ evaluated at the subtraction point) and subtracted forms of the $t$ channel and the above-data part of the $s$-channel integrals. These integrals, and so also $\mathcal{D}_{F}\left(s_{\max } ; a, t\right)$, vary slowly for small $|t|$, just as in the unsubtracted case. The discrepancy method we will employ depends only on this smooth $t$ dependence.

The strategy for determining $F(a, t)$ in the subthreshold region will be to evaluate from experimental data $\mathcal{D}_{F}\left(s_{\text {max }} ; a, t\right)$ in the region $t<0$ by Eqs. (3) and (4), extrapolate it to the subthreshold region [which, by Eq. (1) or its subtracted counterpart, is relatively safe because the nearest singularity of $\mathcal{D}_{F}$ is at $\left.t=4 \mu^{2}\right]$, and then use Eq. (4) again to evaluate $F(a, t)$ in the subthreshold region. We will discuss the procedure more fully later in this section. First, however, we define our choice of invariant amplitudes.

PCAC predictions are often expressed in terms of the amplitudes

$$
D^{( \pm)}(\nu, t)=A^{( \pm)}(\nu, t)+\frac{\nu}{4 m} B^{( \pm)}(\nu, t)
$$

where $A^{( \pm)}$and $B^{( \pm)}$are the standard invariant amplitudes [12]. These amplitudes satisfy the crossing relations $D^{( \pm)}(-\nu, t)= \pm D^{( \pm)}(\nu, t)$. For applications of IDR's we adopt the crossing-symmetric amplitudes $D^{+}$and $\widetilde{D}^{(-)}$ $\equiv D^{(-)} / \nu$.

The IDR Born terms are

$$
\begin{aligned}
& D_{N}^{(+)}=\frac{g^{2}}{4 m} \frac{\left(t_{N}-2 \mu^{2}\right)^{2}}{\left(m^{2}-a\right)\left(t-t_{N}\right)}, \\
& \widetilde{D}_{N}^{(-)}=\frac{g^{2}}{4 m} \frac{t_{N}-2 \mu^{2}}{\left(m^{2}-a\right)\left(t-t_{N}\right)},
\end{aligned}
$$

where $g$, the pion-nucleon coupling constant, is given by $g^{2} / 4 \pi=(2 m / \mu)^{2} f^{2} \approx 13.7$ (i.e., $\left.f^{2} \approx 0.076\right), \quad \nu_{N}=t-2 \mu^{2}$, and $t_{N}=t\left(s=m^{2}, a\right)=\mu^{2}\left(4 m^{2}-\mu^{2}\right) /\left(m^{2}-a\right)$ is the position of the nucleon pole on the curve defined by the path parameter $a$.

Because the pole terms are singular within the subthreshold region, it is desirable to plot the much smoother polesubtracted ("barred") amplitudes $\bar{D}^{(+)}(\nu, t) \equiv D^{(+)}(\nu, t)$ $-D_{N, t}^{(+)}(\nu, t)$ and $\widetilde{\bar{D}}^{(-)}(\nu, t) \equiv \widetilde{D}^{(-)}(\nu, t)-\widetilde{D}_{N, t}^{(-)}(\nu, t)$, where the fixed- $t$ dispersion-theoretic Born terms are

$$
D_{N, t}^{(+)}(\nu, t) \equiv \frac{g^{2}}{m}\left(\frac{\nu_{N}^{2}}{\nu_{N}^{2}-\nu^{2}}\right)=\frac{g^{2}}{4 m} \frac{\left(t-2 \mu^{2}\right)^{2}}{\left(m^{2}-s\right)\left(m^{2}-u\right)}
$$

and

$$
\widetilde{D}_{N, t}^{(-)}(\nu, t) \equiv \frac{g^{2}}{m}\left(\frac{\nu_{N}}{\nu_{N}^{2}-\nu^{2}}\right)=\frac{g^{2}}{4 m} \frac{t-2 \mu^{2}}{\left(m^{2}-s\right)\left(m^{2}-u\right)} .
$$

The "barred" amplitudes are finite everywhere in the subthreshold crescent, independent of whether IDR or fixed- $t$ Born terms are subtracted. The latter are more conventional, however, so it is these that are plotted in our figures. IDR Born terms must, of course, be used in the actual evaluation of the dispersion relations. The two types of Born terms are related by

$$
\begin{gathered}
D_{N}^{(+)}-D_{N, t}^{(+)}=\frac{-g^{2}}{m^{2}-a}\left(\frac{t+t_{N}-4 \mu^{2}}{4 m}\right), \\
\widetilde{D}_{N}^{(-)}-\widetilde{D}_{N, t}^{(-)}=\frac{-g^{2}}{4 m\left(m^{2}-a\right)} .
\end{gathered}
$$

These equations obviate the numerical cancellation of poles in our reconstruction of the barred amplitudes.

Since $\operatorname{Im} F$ can be evaluated in the $s$ channel by the use of experimental phase shifts for $(m+\mu)^{2}<s<s_{\max }$, the data integral $I_{F}\left(s_{\max } ; a, t\right)$ can be computed for all negative values of $a$ and for any (positive or negative) value of $t$. On the other hand the $t$-channel integral requires knowledge of $\operatorname{Im} F$ for the $t$-channel reaction $N \bar{N} \rightarrow \pi \pi$ in the region $t>4 \mu^{2}$. In the region $t>4 m^{2}, \operatorname{Im} F$ is related to the experimental cross section for $N \bar{N} \rightarrow \pi \pi$, but in the interval $4 \mu^{2}<t<4 m^{2}$ this process is unphysical, and a model is needed for the evaluation of $\operatorname{Im} F$.

In the region $4 \mu^{2}<t<16 \mu^{2}, F$ is related to the $\pi \pi$ elastic scattering amplitude for $I=0,1$, or turning it around, knowledge of $F$ will lead to values of these phase shifts. The ability to determine $\pi \pi$ scattering lengths from elastic $\pi N$ scattering data results from extended unitarity [13] which ensures that $\pi N, t$-channel partial-wave amplitudes have the 
same phase as $\pi \pi$ partial-wave amplitudes for $4 \mu^{2}<t$ $<16 \mu^{2}$. Since the lowest partial-wave amplitude in the $t$-channel dominates the invariant amplitude [14] near the $\pi \pi$ threshold at $t=4 \mu^{2}$, the behavior of the $\pi N$ invariant amplitudes at this point can be used to extract $\pi \pi$ scattering lengths [15]. We will make use of this technique to obtain the $a_{00}$ and $a_{11}$ scattering lengths in the next section.

Even though we cannot evaluate $\mathcal{D}_{F}\left(s_{\max } ; a, t\right)$ within the crescent directly from experimental data, there is a standard method which allows us to infer $\mathcal{D}_{F}\left(s_{\max } ; a, t\right)$, and hence $F(a, t)$, within this region. As outlined earlier, the first step is to evaluate the effective discrepancy function within the the $s$-channel physical region $\left[s_{\max } \gg s \geqslant(m+\mu)^{2}, t \leqslant 0\right]$ along a curve of constant $a<0$. Hence, all of the quantities on the right side of Eq. (4) may be determined from known $s$-channel data and the reasonably well-known value of the pion-nucleon coupling constant $g$.

The recent phase-shift analysis SP98 [6] is employed to evaluate the amplitudes $F$ and the data dispersion integral along a curve with fixed $a$ within this region. In some of our calculations the well-known KH80 phase shifts are used above the region of the validity of SP98. For example, calculations performed with $\sqrt{s_{\max }}=4.4 \mathrm{GeV}$ (with SP98 from threshold to $\sqrt{s}=2.0 \mathrm{GeV}$ and $\mathrm{KH} 80$ from 2.0 to $4.4 \mathrm{GeV}$ ) produced subthreshold amplitudes nearly indistinguishable from those which used $\sqrt{s_{\max }}=2.5 \mathrm{GeV}$ (with SP98 alone). This near equality does not imply that the dispersion integrals above $s_{\max }$ are small, but rather it confirms that they are smooth functions of $t$, when $t$ is in the low-energy or crescent region.

The next step is to analytically continue the effective discrepancy function into the crescent region along this curve of fixed $a$. This procedure is relatively safe because, as is seen from its definition and Eq. (1), $\mathcal{D}_{F}$ has no singularities within the crescent. Typical curves, shown in Fig. 1, enter the crescent region at the $s$-channel threshold point at $t=0$ and arrive on the $\nu$ axis at the points $\nu=0, t=t_{-} \equiv \Sigma-2 a$ $-\sqrt{(\Sigma-2 a)^{2}-(4 m \mu)^{2}}$.

As $a$ is varied from 0 to $-\infty$, the $\nu=0$ intersection of the curves moves from $t_{-}=4 \mu^{2}$ to 0 . Thus, by varying $a$, we are able to map the effective discrepancy function throughout the entire crescent region. Once $\mathcal{D}_{F}\left(s_{\max } ; a, t\right)$ has been analytically continued within the crescent, the amplitude $F$ can be reconstructed by rewriting Eq. (4) as

$$
F(a, t)=\mathcal{D}_{F}\left(s_{\max } ; a, t\right)+F_{N}(a, t)+I_{F}\left(s_{\max } ; a, t\right) .
$$

[As shown earlier, $I_{F}\left(s_{\max } ; a, t\right)$ may be calculated from the experimental data for any value $t$ at any value of $a \leqslant 0$.]

\section{SUBTHRESHOLD EXTRAPOLATIONS}

To perform the analytic continuation, $\mathcal{D}_{F}\left(s_{\max } ; a, t\right)$ is expanded in a suitable basis set $\sum_{n=0}^{N} c_{n}(a) \phi_{n}(t)$, where $\phi_{n}(t)$ are analytic functions to be chosen. The coefficients $c_{n}(a)$ are determined for each value of $a$ via a least-squares fit to the known values of $\mathcal{D}_{F}\left(s_{\max } ; a, t\right)$ in the $s$-channel physical region. Typical choices of the basis $\phi_{n}(t)$ are (a) $t^{n}$, (b) $\tau^{n}$, where $\tau \equiv \sqrt{1-t / 4 \mu^{2}}=-i p_{\pi \pi} / \mu$ is proportional to the $\pi \pi$ momentum, and (c) functions obtained by modeling the imaginary part of $F$ by $t$-channel resonance forms $\left(\sigma, \rho, f_{0}\right.$, etc., mesons) [16]. For this work we will adopt method (b), which is simple and flexible; in addition it incorporates the $\pi \pi$ threshold behavior, associated with the branch point at $t=4 \mu^{2}$, which is the nearest singularity. We emphasize that the effective discrepancy function has no nearby $s$-channel singularities; it involves a dispersion integral over the $t$-channel cut running from $4 \mu^{2}$ to $\infty$, and hence an expansion about the $\pi \pi$ threshold in the $\pi \pi$ center-of-mass momentum (or equivalently in $\tau$ ) is a sensible approach. Once contributions from Born terms are removed [15], the functions are expected to be smooth in this region and an expansion in $\tau$ should be adequate. We have used the smallest value of $N$ which gives an acceptable fit to the function $\mathcal{D}_{F}\left(s_{\text {max }} ; a, t\right)$ in the $s$-channel physical region. Usually $N$ $=3$ or 4 is adequate as is explained shortly.

To model the discrepancy function for the amplitude $D^{(-)}$, the term linear in $\tau$ must be omitted. This follows from an examination of the $t$-channel partial-wave expansion [15]. Above the $\pi \pi$ threshold $t=4 \mu^{2}, \tau$ is pure imaginary, and the imaginary part of the discrepancy function and reconstructed amplitude are given entirely by terms with odd powers of $\tau$. The leading term in the $t$-channel partial-wave expansion is a linear combination of the $J=1$ helicity amplitudes $f_{ \pm}^{(1)}\left(p_{\pi \pi}\right)$. Near the $\pi \pi$ threshold, $\operatorname{Im} f_{ \pm}^{(J)}$ is proportional to $p_{\pi \pi}^{2 J+1} \equiv(i \tau \mu)^{2 J+1}$; hence the lowest term of odd power in the expansion is proportional to $\tau^{3}$. Terms with higher $J$ have successively higher powers of $\tau$, and so the term linear in $\tau$ is absent in the expansion. The leading term in the $t$-channel partial-wave expansion of $D^{(+)}$has $J=0$, and so the term proportional to $\tau$ is present in the expansion.

To evaluate the amplitudes along the boundary line $t$ $=0$, we have used a forward dispersion relation. As with the IDR's, it is sufficient to use an unsubtracted form of the discrepancy function:

$$
\mathcal{D}_{F}\left(\nu_{\max } ; \nu\right) \equiv F(\nu)-F_{N, t}(\nu)-I_{F, t}\left(\nu_{\max } ; \nu\right),
$$

where $I_{F, t}$ is defined by

$$
I_{F, t}\left(\nu_{\max } ; \nu\right) \equiv \frac{2}{\pi} \int_{4 m \mu}^{\nu_{\max }} \operatorname{Im} F\left(\nu^{\prime}\right) \frac{\nu^{\prime}}{\nu^{\prime 2}-\nu^{2}} d \nu^{\prime},
$$

$F_{N, t}$ is the appropriate Born term, $\nu_{\max }$ is the upper limit of the validity of the phase-shift analysis, and $F(\nu)$ could be either $D^{(+)}(\nu, t=0)$ or $\widetilde{D}^{(-)}(\nu, t=0)$. These discrepancy functions are very smooth in the low-energy part of the $s$-channel physical region and are excellently fit by polynomials (quadratic) in $\nu^{2}$. Just as with the IDR's, the extrapolated values of $I_{F}\left(\nu_{\max }, \nu\right)$ are combined with Eq. (13) and solved for $F(\nu)$ between $\nu=0$ and the threshold point $\nu$ $=4 m \mu$.

There are three easily identifiable sources of uncertainties in our results. First, there is the uncertainty in the experimentally derived phase shifts and inelasticities used in computing the discrepancy function in the $s$-channel physical region. The other two uncertainties derive from the procedure used in the extrapolations: the choice of $N$, the order of the poly- 


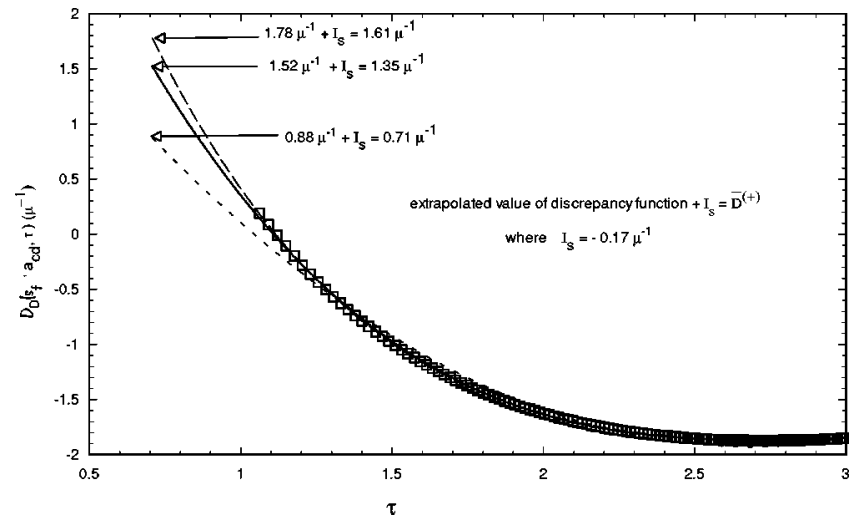

FIG. 2. Discrepancy function for $D^{+}$with $s_{f}=2.5 \mathrm{GeV}^{2}$ and $a$ $=a_{c d} \approx-0.87 \mathrm{GeV}^{2}$. For this value of $a$, the Cheng-Dashen (ca) point occurs at $\tau=1 / \sqrt{2}$, and both IDR and fixed- $t$ Born terms are zero. Extrapolated values for the $\mathrm{CD}$ point are shown for quadratic (short-dashed line), cubic (solid line), and quartic (long-dashed line) polynomials in $\tau$. The boxes represent the discrepancy function in the $s$-channel physical region $(\tau>1)$, evaluated using SP98. A rough estimate of error bars on the latter is $\pm 0.1 \mu^{-1}$. The value of $D^{(+)}$at the CD point is the sum of the extrapolated value of the discrepancy function and $I_{D} \approx-0.17 \mu^{-1}$; see Eq. (12).

nomial in $\tau$, and the choice of the range $s=\left(s_{\text {th }}, s_{f}\right)$ over which the discrepancy function is fit. The corresponding limits on $t$ are $\left(t_{f}, 0\right)$, where $t_{f}(a)=\left[s_{f}\left(\Sigma-s_{f}\right)-\left(m^{2}\right.\right.$ $\left.\left.-\mu^{2}\right)^{2}\right] /\left(s_{f}-a\right)$.

The SAID database gives both global fits (such as SP98) and, at energies where sufficient data exist, single-energy fits. Because the uncertainties of the individual partial-wave amplitudes are strongly correlated, it is difficult to use them in estimating the errors in the corresponding invariant amplitudes and discrepancy functions. We placed crude "error bars" on our reconstructed discrepancy functions by comparing the scatter between the values obtained from singleenergy fits and those obtained from the smooth global fit SP98 [17]. Next, the discrepancy functions (and the corresponding values of $\chi^{2}$ ) are least-squares fit with a sequence of polynomials in $\tau$ of increasing order $N$. From these fits, we select the one with the lowest order, $N=N_{0}$, which gives a satisfactory $\chi^{2} . N_{0}$ is chosen as the smaller of two numbers: the value above which there is no significant improvement in the $\chi^{2}$ or the value for which the $\chi^{2}$ per data point falls below 1 . For both $\bar{D}^{(+)}$and $\bar{D}^{(-)}$, we find $N_{0}=4$, i.e., a cubic, if $s_{f}$ lies in the range $2.0-2.5 \mathrm{GeV}^{2}$. For the smaller value $s_{f}=1.75 \mathrm{GeV}^{2}$, a quadratic is adequate $\left(N_{0}=3\right)$. We generally prefer extrapolations with a larger value of $s_{f}$; the larger intervals serve to better determine the curvature of the discrepancy functions.

Figure 2 illustrates the extrapolation procedure for the case of the $D^{(+)}$amplitude. For this example, we have taken $a=a_{C D}=-m^{2}+\mu^{2} / 2$. In this case the path intersects the point $\left(t=2 \mu^{2}, \nu=0\right)$ at $\tau=1 / \sqrt{2}$. (This is the Cheng-Dashen point, to which we return shortly.) From this figure it is seen that the quadratic fit is inadequate, but the cubic gives an excellent fit in the $s$-channel physical region: $\tau>1$. The

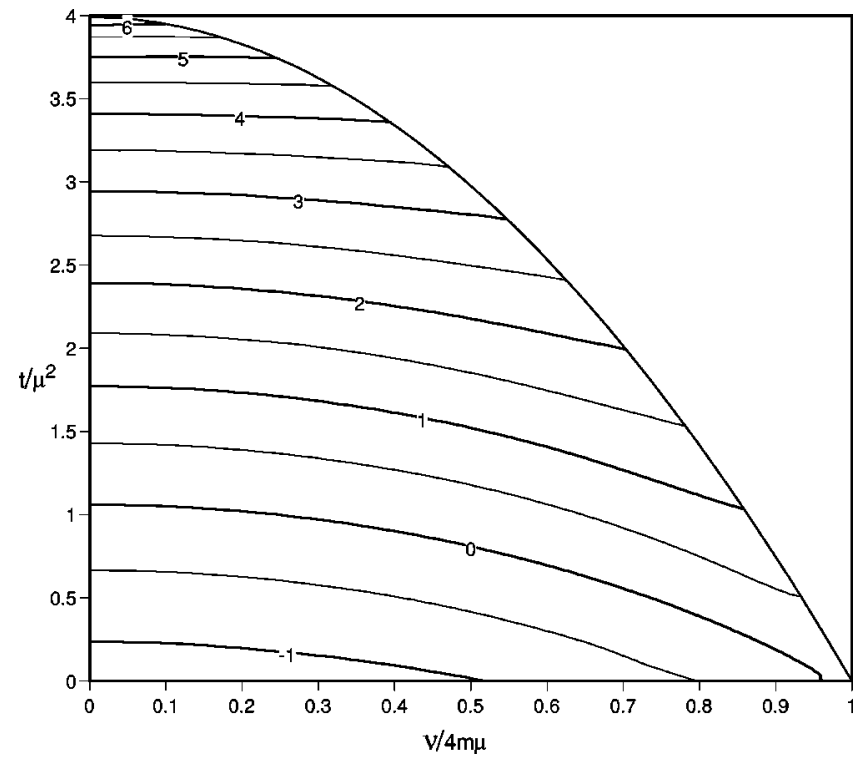

FIG. 3. The isospin-even amplitude $\bar{D}^{(+)}$in units $\mu^{-1}$. The null curve passes near both the Adler point $\left(\nu=0, t / \mu^{2} \equiv 1\right)$ and the $s$-channel threshold point $(\nu / 4 m \mu=1, t=0)$. This plot was computed with $s_{f}=2.5 \mathrm{GeV}^{2}$ and $N_{0}=4$. The uncertainty in these contours is reflected by the error bars given in Fig. 5 .

quartic fit gives no significant improvement of $\chi^{2}$, but it gives us a crude estimate of the error associated with the extrapolation.

We now turn to the results of the calculation of the amplitudes $D^{( \pm)}$. The overall features of the amplitudes are best seen from the contour plots given in Figs. 3 and 4. A convenient parametrization of these amplitudes is given in the Appendix. The contour plots were calculated using $N=4$ (cubic) for both $D^{(+)}$and $D^{(-)}$. The amplitudes are $s u$ crossing symmetric, so only the region with $\nu \geqslant 0$ is shown. Refer to Figs. 5 and 6 for an estimate of the error bars. An interesting

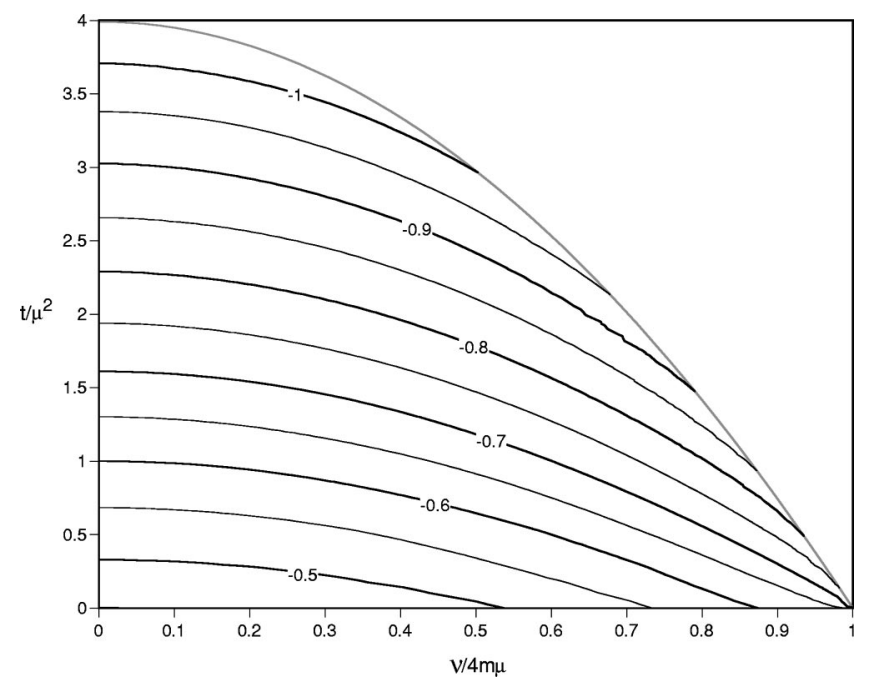

FIG. 4. The isospin-odd amplitude $4 m \overline{\widetilde{D}}^{(-)}$in units $\mu^{-2}$. The Adler-Weisberger point is $\nu=0$ and $t=0$. This plot was computed with $s_{f}=2.5 \mathrm{GeV}^{2}$ and $N_{0}=4$. The uncertainty in these contours is reflected by the error bars given in Fig. 6 . 


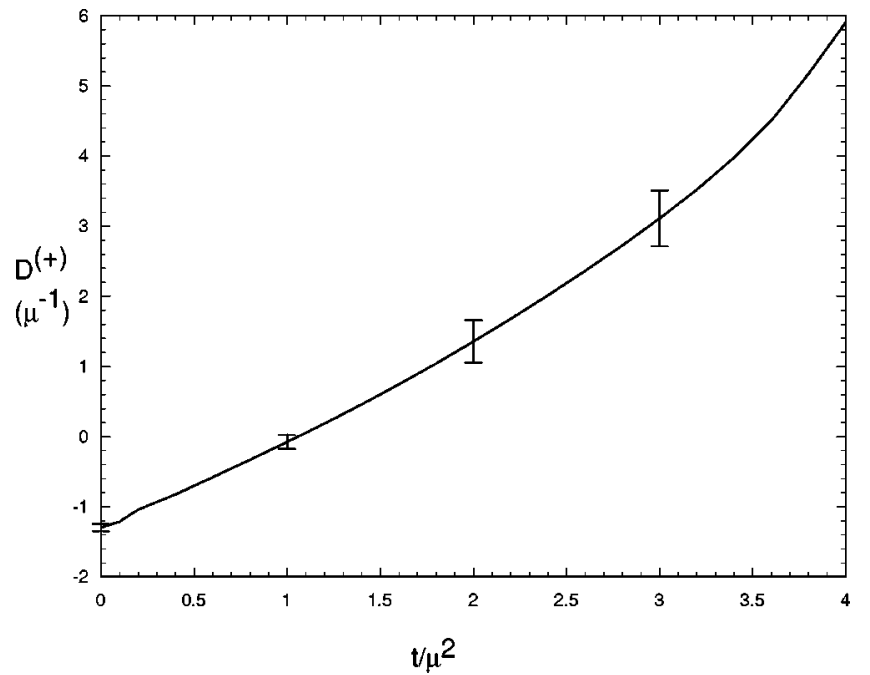

FIG. 5. Amplitude $\bar{D}^{(+)}(0, t)$ along the line $\nu=0$. The parameters are as in Fig. 3.

feature of $\bar{D}^{(+)}$is the null line which passes through the crescent. The Adler consistency condition states $\bar{D}^{(+)}(\nu$ $\left.=0, t=\mu^{2}\right)=0$ (with one soft pion). It is seen from Fig. 3 and Fig. 5 that the zero in our world is at the point $t$ $\approx(1.04 \pm 0.03) \mu^{2}$ instead of at $1.0 \mu^{2}$. At the point $t=\mu^{2}$ the amplitude is very small, $\bar{D}^{(+)}\left(0, \mu^{2}\right) \approx-(0.05 \pm 0.05) \mu^{-1}$. The null line curves downwards with increasing $\nu$, passing though zero at a point very close to threshold. This corresponds to the condition, which follows from current algebra and PCAC, that the isospin-even scattering length $a^{(+)}$is zero in a world of massless pions [3].

For $D^{(+)}$there are (at least) three points of special interest, and the IDR calculation gives all of them within a single consistent analysis. The first is the $s$-channel threshold point $s=(m+\mu)^{2}, t=0$. At this point the isospin-even scattering length is found to be

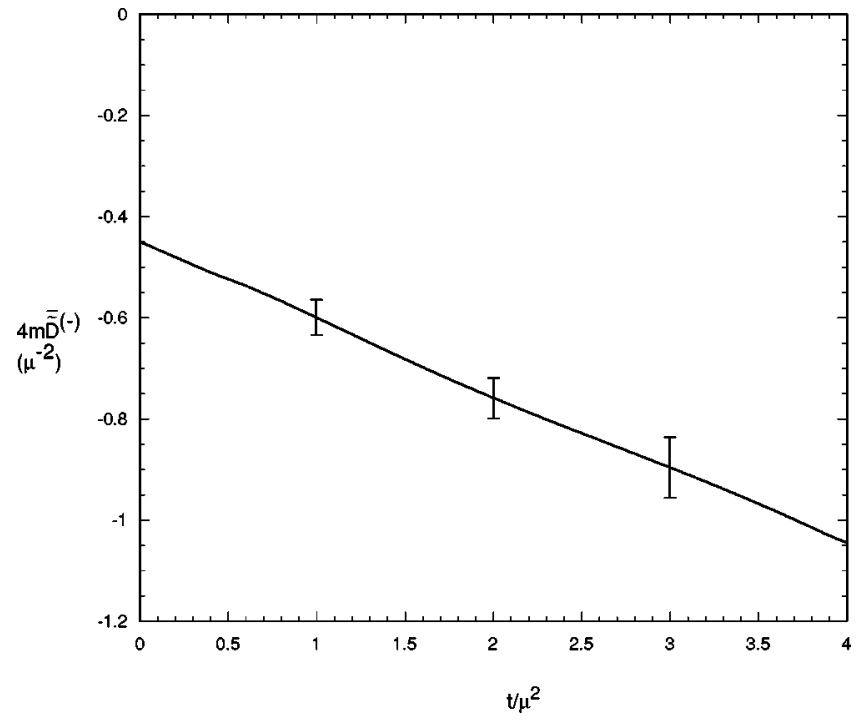

FIG. 6. Amplitude $4 m \overline{\bar{D}}^{(-)}(0, t)$ along the line $\nu=0$. The parameters are as in Fig. 4.

$$
\begin{aligned}
a^{(+)} & =\frac{1}{3}\left(2 a_{3}+a_{1}\right)=\frac{1}{4 \pi(1+\mu / m)} D^{(+)}(4 m \mu, 0) \\
& \approx-0.004 \pm 0.005 \mu^{-1}
\end{aligned}
$$

(note that this is not a "barred" amplitude, so the fixed- $t$ Born term must be included) [18]. This very small number results from the proximity of the null line mentioned in the previous paragraph. The error bar is obtained by comparing the extrapolation to threshold with a broad range of values of the path parameter $a$. The second is the point $\nu=0, t$ $=4 \mu^{2}$ (and hence $a=0$ ) at which the $I=0 \pi \pi$ scattering length $a_{00}$ may be evaluated. Using the method of Ref. [15] with the invariant amplitude $D^{+}$, we obtain the quite reasonable value $a_{00} \approx 0.20 \mu^{-1}$ [19]. That the IDR's give reasonable values of $a_{00}$ and $a^{(+)}$at opposite points on the boundary of the subthreshold crescent adds confidence to the values in the central region. The third special point of interest is the value at the Cheng-Dashen point $\left(\nu=0, t=2 \mu^{2}\right)$ at which the $\sigma$ term may be evaluated [20]. The relationship between the $\sigma$ term and the amplitude at this point is given by the equation [21]

$$
\sigma=\bar{D}^{(+)}\left(\nu=0, t=2 \mu^{2}\right) f_{\pi}^{2} .
$$

The extrapolation to this point is illustrated in Fig. 2. The cubic is the lowest-order polynomial in $\tau$ that gives a satisfactory fit; the corresponding value of $D^{(+)}\left(0,2 \mu^{2}\right)$ is $1.35 \mu^{-1}$, corresponding to $\sigma \approx 83.6 \mathrm{MeV}$. A quartic fit gives $1.61 \mu^{-1}$, or $\sigma \approx 100 \mathrm{MeV}$. Combining these results and those from calculations with smaller values of $s_{f}$, we estimate that $D^{(+)}$at the Cheng-Dashen point is $(1.40$ $\pm 0.25) \mu^{-1}$ (or $\sigma \approx 88 \pm 15 \mathrm{MeV}$ ). This value is somewhat larger than the usually quoted value of about $65 \mathrm{MeV}$. To check that the larger value is not an artifact of our IDR technique, we have repeated our evaluation of the $\sigma$ term using the KH80 amplitudes. Using $s_{f}=1.75$, 2.0, and $2.5 \mathrm{GeV}^{2}$ we obtain $65.2,65.7$, and $65.3 \mathrm{MeV}$, respectively, in agreement with the traditional value. In this case quadratic fits were adequate, but essentially the same results were obtained using cubic fits. We conclude that the large value of the $\sigma$ term is an intrinsic property of the modern SM95 and SP98 phase-shift analyses [22]. At the point $\nu=t=0$ the amplitude is $(-1.30 \pm 0.02) \mu^{-1} \approx-\sigma / f_{\pi}^{2}$, the "antiCheng-Dashen", value, as is expected from the soft-pion theorem for both pions soft. The behavior of $\bar{D}^{(+)}(0, t)$ is shown in Fig. 5. It is seen that the amplitude has a positive slope in $t$ with increasing curvature as $t$ approaches the $\pi \pi \rightarrow N \bar{N}$ pseudothreshold at $4 \mu^{2}$, and $\bar{D}^{(+)}(0,0) \approx$ $-\bar{D}^{(+)}\left(0,2 \mu^{2}\right)[9]$.

The $\widetilde{D}^{(-)}$amplitude is also of great interest. As is seen in Fig. 4 the contour lines of $\overline{\widetilde{D}}^{(-)}$, like those of $\bar{D}^{(+)}$, are also smooth and bend downwards with increasing $\nu$, but the amplitude is negative for all points within the crescent. At the $s$-channel threshold it yields the isospin-odd scattering length 


$$
\begin{aligned}
a^{(-)} & =\frac{1}{3}\left(a_{1}-a_{3}\right)=\frac{4 m \mu}{4 \pi(1+\mu / m)} \widetilde{D}^{(-)}(4 m \mu, 0) \\
& \approx(0.085 \pm 0.030) \mu^{-1}
\end{aligned}
$$

in reasonable agreement with Sigg et al. [23]. At the point $t=4 \mu^{2}$ and $\nu=0$ (i.e., in the limit $a \rightarrow 0$ ) we obtain $p$-wave $\pi \pi$ scattering lengths of $a_{11} \approx(0.035 \pm 0.015) \mu^{-3}$ using the invariant amplitudes $B^{(-)}$and $A^{(-)}$[15]. In addition the point $\nu=0, t=0$ gives a version of the Adler-Weisberger relation [9]:

$4 m \overline{\widetilde{D}}^{(-)}(\nu=0, t=0) \approx\left(1-g_{A}^{2}\right) / 2 f_{\pi}^{2} \approx-(0.690 \pm 0.014) \mu^{-2}$

(Here we have used $g_{A}=1.2670 \pm 0.0035$ and $f_{\pi}=92.42$ $\pm 0.26 \mathrm{MeV}$.) This relation is true for massless pions, but it is seen from Fig. 4 and Fig. 6 that the approximation is approximately satisfied even in our world. In particular, the $t=0$ intercept in Fig. 6 is $(-0.450 \pm 0.025) \mu^{-2}$, not very distant from the value given by the Adler-Weisberger relation, and very close to the values found many years ago in Ref. [9].

\section{ACKNOWLEDGMENTS}

The authors thank Professor R. Jacob and Professor M. Scadron for helpful discussions, and Professor R. Arndt and Professor G. Höhler generously providing us with computerized copies of the SP98 and KH80 phase shifts, and for their encouragement.

\section{APPENDIX}

The contour plots in Figs. 3 and 4 may be reproduced to a good approximation by the following expansions:

$$
\begin{aligned}
\mu \bar{D}^{(+)}(\nu, t) \approx & 6.7436-7.0564 \tau+1.2516 \bar{\nu}^{2}+0.1979 \bar{\nu}^{4} \\
& -0.1087 \tau^{2}-0.0198 \bar{\nu}^{2} \tau^{2}-0.9298 \tau^{3}
\end{aligned}
$$

$$
\begin{aligned}
\mu^{2} 4 m \overline{\widetilde{D}}^{(-)}(\nu, t) \approx & -1.0435\left(1+0.030 \tau^{3}\right)-0.5559 \bar{\nu}^{2} \\
& -0.1690 \bar{\nu}^{4}+0.6135 \tau^{2}+0.4841 \tau^{2} \bar{\nu}^{2},
\end{aligned}
$$

where $\bar{\nu} \equiv \nu / 4 m \mu$ and $\tau=\sqrt{1-t / 4 \mu^{2}}$, which is proportional to the $t$-channel $\pi \pi$ momentum. The variable $\tau$ is used in this expansion instead of the conventional $t$ to model the branch point at $t=4 \mu^{2}$. The large number of digits given in the coefficients is not significant, of course, but is included only to allow accurate reconstruction of the contour plots. These results are obtained from a cubic extrapolation. The forms of the expansions are consistent with the corresponding $t$-channel partial-wave expansions. An estimate of the uncertainty of these amplitudes is given in Figs. 5 and 6. For the parametrization of $\bar{D}^{(+)}(\nu, t)$, it is easy to verify that $a^{(+)}=-0.005 \mu^{-1}, \sigma=84.2 \mathrm{MeV}$, and $a_{00}=0.22 \mu^{-1}$. The amplitude at the "Adler point" $t=\mu^{2}, \nu=0(\tau=\sqrt{3 / 4})$ is $-0.053 \mu^{-1}$ and at the point $t=\nu=0$ is $-1.35 \mu^{-1}$. These are all in reasonably good agreement with the values determined in the body of the paper. For the parametrization of $\mu^{2} 4 m \overline{\widetilde{D}}^{(-)}(\nu, t)$ it follows that $a^{(-)}=0.085 \mu^{-1}$; the amplitude at $t=\nu=0$ is $-0.465 \mu^{-2}$. The latter differs slightly from the value given in the body of the text: $(-0.450$ $\pm 0.025) \mu^{-2}$. The factor $\left(1+0.030 \tau^{3}\right)$ was fixed to make $a_{11} \approx 0.03 \mu^{-3}$. In calculating this scattering volume, the coefficient of the $\tau^{3}$ term is modified by the nucleon Born term as is explained in Ref. [15], Sec. 2.3.
[1] For an extensive review of topics in current algebra and PCAC see V. de Alfaro, S. Fubini, G. Furlan, and S. Rossetti, Currents in Hadron Physics (North-Holland, Amsterdam, 1973). See also the reviews by D. Campbell, in Nuclear Physics with Heavy Ions and Mesons, Proceedings of the Les Houches Summer School of Theoretical Physics, Les Houches, 1977, edited by R. Balian et al. (North Holland, Amsterdam, 1978); M. D. Scadron, Rep. Prog. Phys. 44, 213 (1981).

[2] S. L. Adler, Phys. Rev. 137, B1022 (1965).

[3] S. Weinberg, Phys. Rev. Lett. 17, 616 (1966).

[4] S. L. Adler, Phys. Rev. 140, B736 (1965). See also W. I. Weisberger, ibid. 143, 1302 (1966).

[5] The boundaries of the crescent in the $(\nu, t)$ basis are the line $t=0$ and the hyperbola $(t-\Sigma)^{2}-\nu^{2}=4\left(m^{2}-\mu^{2}\right)^{2}$, where $\Sigma$ $=2 m^{2}+2 \mu^{2}, m$ is the proton's mass, and $\mu$ is the charged pion's mass. The hyperbola corresponds to $a=0$, where $a$ is the IDR path parameter defined below.

[6] VPI phase-shift analysis SP98, courtesy of Professor R. Arndt. Internet access is through http://said.phys.vt.edu

[7] G. E. Hite, R. J. Jacob, and F. Steiner, Phys. Rev. D 6, 3333 (1973).
[8] Some preliminary results using the earlier VPI phase-shift analysis SM95 were given in W. Kaufmann, G. Hite, and R. Jacob, $\pi N$ Newslett. 13, 16 (1997).

[9] G. E. Hite, R. J. Jacob, and M. D. Scadron, Phys. Rev. D 14, 1306 (1976). Over the years this region has also been explored by use of other dispersion relations. See Ref. [12] for a detailed review up to the early 1980s. Later work on evaluations of the sigma term may be traced from M. E. Sainio, Chiral Dynamics: Theory and Experiment, edited by A. M. Berstein and B. R. Holstein (Springer, Berlin, 1994), p. 212.

[10] Pioneering work in mapping this region was performed long ago by G. Höhler, H. P. Jacob, and R. Strauss, Nucl. Phys. B39, 237 (1972) and by H. Nielsen and G. C. Oades, ibid. B72, 310 (1974).

[11] This expression is related to the Kibble function for the $\pi N$ system: $\phi(s, t, u)=t\left[s u-\left(m^{2}-\mu^{2}\right)^{2}\right]$. The physical boundary is given by $\phi(s, t, u)=0$. See T. W. B. Kibble, Phys. Rev. 117, 1159 (1960).

[12] G. Höhler, in $\pi N$ Scattering: Phenomenological Analyses, edited by H. Schopper, Landolt-Börnstein, New Series, Group 1, 
Vol. 9b, Pt. 2 (Springer, Berlin, 1983). This handbook is an extremely useful compendium of information about the $\pi N$ system. This book defines $\nu=(s-u) / 4 m$, which differs by the factor of $4 \mathrm{~m}$ from the value used in the present article.

[13] S. Mandelstam, Phys. Rev. Lett. 4, 84 (1960).

[14] W. R. Frazer and J. R. Fulco, Phys. Rev. 117, 1603 (1960). The idea of determining $\pi \pi$ scattering lengths from $\pi N$ scattering data is very old; see the historical summary in Sec. 4.1 of B. R. Martin, D. Morgan, and G. Shaw, Pion-Pion Interactions in Particle Physics (Academic Press, London, 1976). This reference also traces the early development of the discrepancy function.

[15] R. Müller, G. E. Hite, and R. J. Jacob, Z. Phys. C 8, 199 (1981); G. E. Hite and R. J. Jacob, Nucl. Phys. B134, 291 (1978).

[16] See, for example, Richard J. Jacob and Gerald E. Hite, Phys. Rev. D 11, 2466 (1975); E. Borie and F. Kaiser, Nucl. Phys. B126, 173 (1977).

[17] We thank Prof. R. Arndt for a discussion on this point.
[18] The IDR method is a good technique for the evaluation of the $\pi N$ scattering lengths $a^{( \pm)}$because every fixed- $a$ path passes through the $s$-channel physical threshold. Each value of the path parameter gives a different extrapolation of the amplitudes to threshold. The variation in values of the threshold amplitude using these different paths is a measure of the uncertainty in the predicted scattering length.

[19] This is in excellent agreement with the recent modelindependent value $a_{00}=(0.206 \pm 0.013) \mu^{-1}$, given by M. Kermani et al., $\pi N$ Newslett. 13, 27 (1997).

[20] D. C. Moir, R. J. Jacob, and G. E. Hite, Nucl. Phys. B103, 477 (1976).

[21] See T. P. Cheng and R. Dashen, Phys. Rev. Lett. 26, 574 (1971) and the reviews in Ref. [1].

[22] The larger value is in accordance with our preliminary result [8] and also the work of M. Pavan and R. Arndt, $\pi N$ Newslett. 13, 165 (1997), which is also based on the VPI phase-shift analysis.

[23] D. Sigg et al., Phys. Rev. Lett. 75, 3245 (1995). 